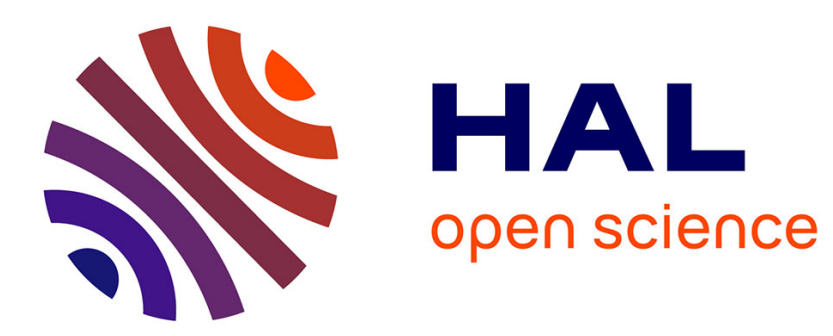

\title{
Le Droit Parlementaire: clé de la crise du Gouvernement Conte I
}

\author{
Adriano Evangelisti
}

\section{To cite this version:}

Adriano Evangelisti. Le Droit Parlementaire: clé de la crise du Gouvernement Conte I. Civitas Europa, 2019, 43. halshs-02422912

\section{HAL Id: halshs-02422912 \\ https://shs.hal.science/halshs-02422912}

Submitted on 23 Dec 2019

HAL is a multi-disciplinary open access archive for the deposit and dissemination of scientific research documents, whether they are published or not. The documents may come from teaching and research institutions in France or abroad, or from public or private research centers.
L'archive ouverte pluridisciplinaire HAL, est destinée au dépôt et à la diffusion de documents scientifiques de niveau recherche, publiés ou non, émanant des établissements d'enseignement et de recherche français ou étrangers, des laboratoires publics ou privés. 
ADRIANO EVANGELISTI Doctorant UMR 7318, ILF/GERCJ, ED 67, Faculté de Droit d'Aix en P.ce

Suite à la séance plénière du Sénat, le 7 août dernier, le Gouvernement Conte $I$ est entré en crise. Cette définition reste néanmoins assez approximative car d'un point de vue juridique celle-ci n'a pas été parlementarisée.

En effet le 7 août, dernier jour de séance pour le Sénat avant la fin de la session parlementaire, ont été votées les motions sur la TAV (acronyme indiquant la Train Haute Vitesse Turin-Lyon). Sur ce projet subsistait une divergence entre les forces politiques du gouvernement : la Ligue de Matteo Salvini étant favorable à la réalisation du projet, le $M 5^{*}$ fortement opposé.

6 motions ont été déposées: 2 défavorables ( 1 du M5*, l'autre d'un parti de gauche faisant partie du Groupe Mixte au Sénat) à la réalisation de ce projet, 4 favorables qui demandaient au Gouvernement de prendre toutes les mesures nécessaires à la mise en œuvre définitive de ce projet. Lors du vote les divisions politiques présentes au sein de la majorité gouvernementale se sont manifestées avec deux avis opposés: le Vice-Ministre de l'Économie Garavaglia (Ligue) était pour l'approbation des motions présentées par les groupes d'opposition (motions 1-00153, 1-000156, 1000157, 1-000162), le sous-secrétaire du Gouvernement Santangelo (M5*) opposé à la TAV, contraint par l'absence d'une majorité s'en remettait à l'Assembléel.

Le 4 motions favorables à la réalisation du projet étaient approuvées, en revanche les deux autres et surtout celle du M5* (1-00152), qui demandait au Parlement de prendre toutes les initiatives nécessaires pour cesser toutes les activités liées à la mise en ouvre du projet et d'affecter des ressources économiques à d'autres projets ${ }^{2}$, étaient rejetées.

La motion de M5* engageait le Parlement et non pas le gouvernement. Il ne s'agit pas d'un simple détail, car normalement le dispositif final d'une motion termine avec la formule classique «la motion engage le Gouvernement[...]». La motion de M5* constitue un cas assez singulier et atypique de motion, en effet elle ne souhaitait pas engager le Gouvernement car son Premier Ministre Conte, proche aux M5*, s'était engagé pour réaliser définitivement le projet. Une motion qui engageait le gouvernement à ne pas réaliser le projet TAV aurait été en contradiction avec la ligne politique de l'exécutif et aurait pu entraîner l'ouverture d'une crise de gouvernement.

Suite à l'approbation des motions la Ligue, au vu des divergences trop importantes présentes dans le Gouvernement, décida d'ouvrir la crise de gouvernement au moyen d'une motion de censure ${ }^{3}$ (ex.article 94, dernier alinéa de la constitution). Néanmoins toutes les crises (parlementaires, extraparlementaires) généralement ${ }^{4}$ pour être effectives d'un point de vue juridique nécessitent une

${ }^{1}$ Il s'agit d'une formule courante utilisée dans le droit parlementaire italien pendant l'approbation d'amendements, sousamendements, articles d'une loi, motions ou résolutions lorsque le Gouvernement doit donner un avis sur les textes qui sont mis au vote. Quand le Gouvernement sait que sur un texte mis au vote la majorité risque d'être battue, il décide de s'en remettre à la décision de l'Assemblée. Autrement dit, l'exécutif sait qu'il n'aura pas la majorité et pour n'être pas battue (il le saurait s'il donnait un avis démenti par un vote de l'Assemblée opposé aux choix du gouvernement) il décide de s'abstenir et de laisser décider l'Assemblée (De iure le Gouvernement n'est pas battu car il n'a fourni aucun avis, De facto et politiquement la majorité soutenant le gouvernement est battue). Le fait de s'en remettre à l'Assemblée peut constituer un prélude à une crise de gouvernement, cela dépend de l'importance politique du texte en discussion car souvent on utilise aussi cet instrument sur des aspects techniques d'un texte en discussion. Concernant les motions sur la $\mathrm{TAV}$, on constate que la position du sous-secrétaire de s'en remettre à l'Assembée a été le prélude d'une crise de gouvernement.

${ }^{2}$ Point 1) et 2) de la partie finale de la motion. Cfr http://www.senato.it/service/PDF/PDFServer/BGT/1123213.pdf

${ }^{3} \mathrm{Si}$ la motion est effectivement votée il s'agirait de la première fois dans l'histoire républicaine italienne qu'une motion de censure est présentée et votée par un parti de la majorité contre son propre gouvernement.

${ }^{4}$ Les crises peuvent surgir pour des désaccords au sein de la majorité qui rendent impossible l'action du gouvernement. Dans ces circonstances le Premier Ministre démissionne sans passer par un vote du Parlement (crise extraparlementaire). Néanmoins le désaccord entre les forces politiques qui soutiennent l'exécutif peut se manifester par un vote du Parlement (crise extra-parlementaire). La majorité des crises en Italie ont été d'ordre extra-parlementaire, seulement les deux crises du Gouvernement Prodi (1998 et 2008) ont été parlementarisées. Cette tendance est due à la 
parlementarisation: le Premier Ministre se rend aux assemblées pour constater que sa majorité n'existe plus et, éventuellement après un vote, remet sa démission au Président de la République.

Un autre acte qui généralement constitue un acte préalable à une crise de gouvernement consiste dans la démission des Ministres: en effet le retrait des Ministres d'un parti politique en rupture avec l'autre composante de la majorité, est une sorte de pré-crise parlementaire ou une antichambre de la parlementarisation de la crise.

Néanmoins l'actuelle crise politique qui s'est récemment ouverte présente des aspects singuliers car le chef de la Ligue Matteo Salvini, Ministre de l'Intérieur, n'a pas présenté la démission des 7 Ministres présents au sein de l'exécutif Conte I.

On peut ainsi parler d'une crise qui ne s'est pas concrétisée au sein des Assemblées parlementaires, une sorte de pré-crise de gouvernement.

Dans ces circonstances les étapes de cette crise sont déterminantes et notamment deux aspects juridiques, d'ordre technique et relevant du droit parlementaire, revêtent une importance décisive pour la résolution de la crise:

la définition du Calendrier des Travaux du Sénat (art.55 du règlement) suite au dépôt d'une motion de censure par la Ligue ( I ) et aussi le calendrier des travaux établi par Conférence des Présidents de la Chambre des députés ( II ).

\section{I- La définition du Calendrier des Travaux du Sénat}

Il est important de souligner que la crise a été ouverte le dernier jour de séance du Sénat, après que ce dernier avait établi le Calendrier des Travaux pour le mois de septembre. Cette crise est ainsi intervenue lorsque les chambres ne tenaient plus de session, une première dans l'histoire républicaine italienne. L'effet a été celui d'une nouvelle redéfinition du calendrier des travaux par la Conférence des Présidents, surtout à la lueur de la motion de censure déposée par la Ligue au Sénat. Concernant la définition de ce Calendrier, qui a normalement une nature mensuelle, ce dernier est approuvé à l'unanimité par la Conférence, dans cette circonstance le calendrier est définitif. Néanmoins en absence d'unanimité, le calendrier est provisoire et peut faire l'objet de modification en Assemblée de la part d'un sénateur par groupe (art.55 alinéa 3 règlement). En présence de modifications, a lieu un débat sur la modification $d u$ calendrier : un orateur par groupe peut intervenir pour une durée maximale de 10 minutes. A l'issue du débat, un vote est organisé à main levée. Le calendrier devient alors définitif.

La Conférence des Présidents, réunit lundi 12 août, en l'absence de l'unanimité approuva un calendrier des travaux provisoires, qui a fait l'objet d'un vote de la part de l'Assemblée en raison de demandes de modifications.

Deux hypothèses de Calendrier des Travaux ont été alors débattues en séance : une (de la Ligue) qui demandait de mettre à l'ordre du jour de la séance du 14 août la discussion et la votation de la motion de censure déposée, l'autre (des oppositions et du M5*) de convoquer le Sénat le 20 août pour des communications du Premier Ministre Conte.

A l'issue du vote a été approuvée la deuxième avec 161 voix pour soit la majorité absolue des membres du Sénat.

La proposition de la Ligue, souhaitant parlementariser le plus vite possible la crise, incluait un autre point : afin de dissoudre le Parlement et de convoquer des élections anticipées, la Ligue manifestait la volonté de voter la loi constitutionnelle réduisant les nombre des parlementaires ${ }^{5}$, qui avait fait

conduite des partis politiques qui bien souvent ne considèrent pas opportun de rendre officielle par un vote parlementaire la crise de l'exécutif. Toutefois, si on interprète de manière correcte les dispositions constitutionnelles (notamment l'article 94 de la constitution), chaque crise devrait être parlementarisée. Cette tendance majoritaire à ne pas vouloir parlementariser les crises témoignent de la volonté des forces politiques d'en finir avec l'expérience d'un gouvernement mais surtout la faible prise en compte du rôle du Parlement, qui est tenu de plus en plus, à l'écart des crises gouvernementales.

${ }^{5}$ Il s'agit du texte de loi constitutionnelle numéro 214-515-805 $\mathrm{B}$, qui réduit les parlementaires à 600 (400 députés, contre $630 ; 200$ sénateurs, par rapport aux 315 prévus). 
l'objet de trois lectures et nécessitait une dernière lecture à la Chambre des députés (conformément à la procédure prévue par l'article 138, alinéa 1 de la Constitution), inscrite à l'ordre du jour de la séance du 9 septembre 2019.

La Ligue proposait d'anticiper le vote de cette loi en modifiant le Calendrier des Travaux, établi au préalable par la Conférence des Présidents de la Chambre des députés avant l'ouverture de la crise de gouvernement.

Le schéma de la Ligue consistait ainsi en un échange politique : vote anticipé de la loi constitutionnelle réduisant les parlementaires et en contrepartie élections anticipées. S'agissant de la 4ème et dernière lecture de la loi constitutionnelle, selon le Ministre de l'intérieur Salvini «[...]Une fois que cette loi a été votée, pour des raisons de dignité, d'honnêteté et de cohérence, nous pouvons dissoudre le Parlement et passer immédiatement aux élections anticipées [...] $]^{6} 》$.

Au-delà de la stratégie politique, cette déclaration soulève de nombreuses interrogations d'un point de vue juridique dont la plus importante est la suivante : peut-on voter, en dernière lecture, une loi constitutionnelle réduisant le nombre des parlementaires et dissoudre après le Parlement pour des nouvelles élections?

La réponse à cette question réside dans les alinéas 2 et 3 de l'article 138 de la constitution lesquels, concernant les lois constitutionnelles, prévoient que "Ces lois sont soumises à un référendum populaire lorsque, dans les trois mois, suivant leur publication, un cinquième de l'une des deux chambre, ou cinq cent mille électeurs ou Cinq Conseils Régionaux en font la demande[...]» et «Il n'y a pas lieu à un référendum si la loi a été adoptée au second tour de scrutin par chacune des deux chambres à la majorité de deux tiers de ses membres».

La loi constitutionnelle en question est à sa dernière lecture avant l'éventuel référendum. En effet celui-ci n'a pas lieu seulement si la loi lors de la deuxième délibération a été votée à la majorité des suffrages valables, dans cette circonstance la loi n'est même pas promulguée (fin de l'alinéa 2 article 138). Lorsque la loi obtient la majorité des suffrages le référendum n'est pas automatique car la loi est promulguée au Journal Officiel, mais la demande de référendum doit être faite dans les 3 mois par 1/5 parlementaires (126 députés, 64 sénateurs), 500.000 électeurs ou 5 Conseils Régionaux.

Si dans ce délai n'est faite aucune demande, la loi constitutionnelle est promulguée et entre en vigueur.

En revanche s'il y a une demande, un référendum est organisé et la loi sera promulguée si elle est approuvée par la majorité des suffrages valables (contrairement au référendum abrogatif, le référendum constitutionnel ne requiert aucun quorum de validité).

$\mathrm{Au}$ vu du contenu de l'article 138 il ne serait pas possible d'approuver une loi constitutionnelle réduisant le nombre des parlementaires, dissoudre le Parlement et organiser dans la législature suivante le référendum constitutionnel. En effet une dissolution immédiate du Parlement juste après avoir voté la loi constitutionnelle ne permettrait pas aux parlementaires de pouvoir demander un référendum dans les 3 mois qui suivent la publication de la loi constitutionnelle.

Il est possible de citer quand même un précédent: la loi constitutionnelle votée le 16 novembre 2005, soumise au référendum en juin 2006, juste après les élections d'avril 2006. Cette loi constitue un exemple de loi votée avant la dissolution du Parlement et soumise au référendum juste après les élections politiques. Ce précédent pourrait laisser penser qu'il n'y aurait pas d'obstacle. Toutefois il faut préciser que si les élections politiques avaient eu lieu avant le référendum, le droit des parlementaires de pouvoir demander le référendum dans les 3 mois suivants la promulgation de la loi avait été préservé : le décret de dissolution du Parlement fut promulgué en février 2006, juste 3 mois après le dernier vote du Parlement.

Pour ces raisons, d'un point de vue constitutionnel il paraît tout à fait impossible juridiquement de suivre le schéma proposé par Salvini. Si son article 4 prévoit qu'en cas d'approbation au référendum la loi entre en vigueur à compter de la législature suivante même en cas de dissolution du Parlement, ces dispositions doivent tenir compte des dispositions prévues à l'article 138.

En outre pour que la loi soit effective il serait nécessaire d'effectuer un redécoupage des circonscriptions électorales en raison de la réduction des parlementaires. Cet œuvre de redécoupage

$\overline{{ }^{6} \text { Compte rendu de la séance du Sénat }}$ du 13/08/2019,http://www.senato.it/service/PDF/PDFServer/BGT/1123280.pdf, site consulté le 14 août 2019. 
nécessiterait entre 60 et 90 jours.

Cet aspect technique additionné aux 3 mois nécessaires pour organiser le référendum constitutionnel aurait la conséquence d'allonger les délais nécessaires. Si aucune demande de référendum était faite dans les 3 mois suivants la publication de la loi, il faudrait toutefois redessiner les circonscriptions électorales, la loi approuvée au référendum serait applicable à compter de février 2020. En revanche s'il y avait une demande de référendum, les délais seraient allongés davantage, vu que pour la tenue du référendum doivent s'écouler 7 mois et 10 jours à compter de la dernière délibération parlementaire; la loi approuvée ne serait pas applicable avant juin 2020.

En tout état de cause, l'approbation de la loi constitutionnelle juste avant la dissolution du Parlement, sans que les 3 mois pour la demande de référendum soient passés, entraînerait l'inapplication sine die du texte, car il constituerait un détournement de la procédure prévue par l'article 138.

Au vu de la situation qui s'est produite, deux solutions sont possibles :

- voter la loi constitutionnelle et suivre les procédures prévues par l'article 138, dans ce cas l'entrée en vigueur pourrait intervenir à compter d'avril 2020 ;

- ne pas voter la loi constitutionnelle et organiser de nouvelles élections, laissant inchangé le nombre des parlementaires (945, 630 députés et 315 sénateurs), laissant à un futur législateur constitutionnel le soin de les réduire.

Enfin, deux autres problèmes d'ordre institutionnel peuvent se poser.

En premier lieu, si la réforme est votée en dernière lecture elle sera soumise au référendum si l'un des sujets mentionnés préalablement (alinéa 2 article 138) en fait la demande. Les élections anticipées auraient lieu avant le référendum et les électeurs éliraient le Parlement avec les effectifs prévus en Constitution. Même dans la circonstance où la loi constitutionnelle est approuvée par référendum, quelle serait la légitimité d'un Parlement avec un nombre de parlementaires qui aurait été réduit par le peuple au référendum? Le Parlement serait quasi illégitime car sa composition actuelle entrerait en conflit avec les effectifs réduits introduits par la nouvelle révision.

En deuxième lieu, est-ce qu'il est opportun que le Parlement approuve une révision de la Constitution comme dernier acte qui clôture la législature? Les révisions constitutionnelles constituent un des actes les plus importants votés par un Parlement, pour cette raison le Parlement doit être pleinement dans l'exercice de ses fonctions et de sa légitimité politique. La dissolution est le résultat d'une rupture entre les forces majoritaires et l'impossibilité à former une nouvelle majorité soutenant un exécutif. Pour cette raison il serait tout à fait curieux que le Parlement approuve une révision constitutionnelle et qu'il soit dissout immédiatement après.

Face à une telle situation, on peut s'interroger si le Président de la République pourrait exercer son pouvoir de renvoi (article 74 alinéa 1 de la Constitution) même à un loi constitutionnelle? La Constitution est muette sur ce point, mais il est vrai qu'on pourrait admettre son éventuel pouvoir de renvoi face à une situation de détournement de procédures à la Constitution. En effet l'article 138, alinéa 2 prévoit la possibilité de ne pas promulguer une loi constitutionnelle lorsqu'elle est approuvée à la majorité simple. Mutatis Mutandis par une interprétation extensive, on pourrait concevoir la possibilité pour le Président de ne pas promulguer une loi constitutionnelle dans une circonstance qui méconnaîtrait et porterait atteinte aux droits des parlementaires de demander le référendum dans les 3 mois suivants sa promulgation (alinéa 2 art.138 constitution).

\section{II- Le Calendrier des Travaux établie par la Conférence des Présidents de la Chambre des députés}

Le même jour où le Sénat s'est réuni pour établir son Calendrier des Travaux, se réunissait aussi la Conférence des Présidents pour (ré)établir son Calendrier des Travaux en raison de la crise politique. 
Par rapport au Sénat, à la Chambre des députés le calendrier des travaux devient définitif lorsqu'il est approuvé par les $3 / 4$ des Présidents des groupes présents en Conférence des Présidents (article 24, alinéa 2 du règlement de la Chambre des députés). En absence de ce quorum, c'est le Président de la Chambre des députés qui établit le calendrier des travaux (alinéa 3 du même article 24).

La Conférence des Présidents de la Chambre des députés réunit le 13 août approuva à l'unanimité le calendrier des travaux comme suit :

- le 21 août à 15 h communications du Premier Ministre;

- le 22 août à $16 \mathrm{~h}$ la votation finale sur la loi constitutionnelle réduisant le nombre des parlementaires.

On peut constater que les calendriers des travaux arrêtés par les deux Assemblées sont intimement liés.

Car le 20 août le Sénat a inscrit les communications du Premier Ministre qui seront faites aussi le lendemain à la Chambre des députés. A l'issue de ces communications pourront être présentées des résolutions, soit des actes législatifs comparables aux motions pouvant clôturer un débat après la votation de motions ou être présentés à l'issue des communications rendues par le Gouvernement. La discussion et votation de la réduction du nombre des parlementaires interviendrait seulement le 22, après les communications rendues par le Premier Ministre.

Il résulte que la séance du 22 à une portée hypothétique, liée aux séances précédentes des 21 et 22. En effet lors de ces communications le Premier Ministre, face au débat qui suivrait, pourrait décider de suspendre la séance et de démissionner, sans attendre la mise aux voix des résolutions. Dans cette circonstance il serait assez compliqué de discuter et surtout de voter le lendemain une loi constitutionnelle en présence d'un gouvernement démissionnaire. La démission de ce dernier aurait un effet suspensif sur la séance du 22, la parlementarisation de la crise rendrait nécessaire des consultations organisées par le Président de la République. En revanche si les résolutions mises aux voix soutenant le gouvernement ne sont pas rejetées, le Gouvernement ne serait pas contraint à démissionner et pourrait avoir lieu le 22 la discussion sur la loi constitutionnelle réduisant les parlementaires. Néanmoins dans cette circonstance, il ne pourrait y avoir des élections immédiates pour les raisons vues précédemment.

Un doute peut surgir quant à la motion de censure déposée. Celle-ci n'a pas été insérée dans le calendrier des travaux de la Séance du Sénat et de la Chambre des députés. Néanmoins, les signataires n'ont pas manifesté l'intention de la retirer, au contraire ils ont affirmé leur volonté de la discuter le 21 au Sénat. Cette possibilité semble assez complexe, vu que le Calendrier des Travaux approuvé a une portée définitive. A moins que la Conférence des Présidents décide d'inclure d'autres points à l'ordre du jour de la séance après les communications du Gouvernement à 15h. Une possibilité pourrait être celle d'inscrire, immédiatement après les communications, la discussion de la motion de censure. Cet indice pourrait être fourni par le site du Sénat car avant la séance de mardi à $15 \mathrm{~h}$ est prévue une convocation de la Conférence des Présidents à $14 \mathrm{~h} 30^{7}$, celle-ci pourrait décider d'ajouter ladite motion à l'ordre du jour.

Au-delà de ces réflexions, on constate que la portée de ce calendrier des travaux revêt une signification cruciale. Ce dernier traduit au mieux la volonté des forces politiques, dans l'attente de la parlementarisation de la crise, le calendrier des travaux a fourni les étapes éventuelles et possibles pour pouvoir résoudre la crise : démission $d u$ Gouvernement avant le vote des résolutions, démission après le vote des résolutions en raison d'absence de majorité soutenant l'exécutif, démission après le vote des résolutions car une nouvelle majorité s'est formée, retrait de la motion de censure avec une résolution définitive de la crise, inscription de la motion de censure après les communications et démission après son éventuelle approbation ou rejet ${ }^{8}$.

${ }^{7}$ Sur le site du Sénat italien (15 août 2019) concernant les travaux de séances est écrit «L'Assemblée de Palazzo Madama est convoquée pour les communications du Président du Conseil le mardi 20 août à 15 heures. Le même jour, la Conférence des présidents des groupes est prévue à $14 \mathrm{~h} 30$ à la Sala Pannini.https://www.senato.it/home, site consulté le 15 août 2019.

${ }^{8}$ Même en cas de rejet de la motion de censure, le Gouvernement serait contraint de démissionner. Un tel rejet de la motion de censure serait possible avec le soutien des oppositions. Néanmoins en présence des groupes d'opposition qui 


\section{CONSIDÉRATIONS FINALES}

Lors de la séance du 21 août au Sénat la crise a été enfin parlementarisée: le Premier Ministre a rendu ses communications, pendant un long débat dont la durée a été arrêté par la Conférence des Présidents de $3 \mathrm{~h}$ et 45 minutes, à l'issue duquel il a demandé une suspension de séance pour présenter sa démission au Président de la République. Il y a eu ainsi une parlementarisation de la crise de facto, qui ne s'est pas concrétisée de iure par un vote parlementaire de censure du gouvernent ${ }^{9}$.

On constate donc comme le calendrier des travaux arrêtés par les Assemblées parlementaires a été déterminant pour cette crise et notamment celui du Sénat qui avait une portée primordiale par rapport à celui de la Chambre des députés ce dernier ayant une portée incidente. En effet l'épicentre de cette crise était se situait au Sénat : en fonction de ce qui se serait passé dans cette assemblée, le calendrier prévu pour le lendemain à la Chambre des députés aurait été inutile. C'est ce qui s'est passé dans les faits vu la démission du Premier Ministre Conte après le débat du Sénat, cette dernière a entrainé un effet préjudiciable sur le calendrier des travaux de la Chambre des députés rendant inutiles les communications du Premier Ministre prévues le 21 août et laissant en suspension le vote sur la loi constitutionnelle réduisant le nombre des parlementaires.

$\mathrm{Au}$ vu de la crise ouverte on peut observer aussi que le calendrier des consultations, prévues les 21 et 22 août $^{10}$, témoigne d'une volonté présidentielle de résoudre assez rapidement la crise de gouvernement, évitant une répétition d'une situation semblable à celle des élections du 4 mars 2018 où 89 jours furent nécessaires pour former le Gouvernement Conte I.

L'approche mise en place par le Président présente des aspects différents de celles suivies au début de la XVIIIème législature. A l'époque, les procédés mis en place furent différents, complexes $(2$ consultations ouvertes à tous les groupes parlementaires, 2 mandats exploratifs, 1 tour de consultations restreintes aux deux seuls groupes de la Ligue et Cinq Étoiles, le mandat attribué à Cottarelli pour former un gouvernement électoral conduisant le pays à des élections législatives anticipées, les 2 mandats attribués à Giuseppe Conte pour former le Gouvernement) par rapport à ceux mis en place dans la crise du Gouvernement Conte I. Au début de la législature les procédés témoignent d'une volonté présidentielle de former un gouvernement, alors que pendant la crise du gouvernement Conte l'intention est celle de résoudre rapidement la crise de gouvernement. Pour

votent contre une motion de censure et une partie des groupes soutenant le gouvernement qui votent en sa faveur, la démission du Premier Ministre serait nécessaire pour un remaniement de l'exécutif, vu qu'il serait soutenu par une nouvelle majorité parlementaire.

${ }^{9}$ Lors de la séance du 21 août, il y a eu un débat parlementaire général après les communications du Premier Ministre. A la fin de celui-ci aucune résolution ni motion de censure n'a été présentée, d'ailleurs lors de la séance plénière la Ligue a retiré sa motion de censure qui à ce jour sic n'est pas consultable. Le retrait de celle-ci s'est rendu nécessaire pour des exigences purement politique et non juridique vu que le Premier Ministre au début de la séance avait manifesté son intention irrévocable de démissionner. On constate comme pour la première fois dans l'histoire républicaine italienne, une motion de censure a été annoncée à la presse et n'a pas fait l'objet d'un dépôt, vu qu'il est impossible de pouvoir consulter sur le site du Sénat ladite motion. Une situation totalement rocambolesque qui peut légitimement faire douter de son réel dépôt. En effet la motion a été annoncée, elle aurait été déposée comme l'on affirmait certains sénateurs signataires de la Ligue, elle est néanmoins non consultable et à plusieurs reprises ses potentiels et éventuels signataires ont manifesté l'intention de pouvoir la retirer. Il faudrait parler peut-être d'un genre de pré-motion de censure ce qui constituerait une absurdité et un détournement des dispositions constitutionnelles prévues à l'article 94 régissant les procédures d'approbation et de présentation. On constate hélas comme la conduite suivie témoigne d'une volonté de détourner les procédures prévue par la Constitution et de marginaliser l'activité du Parlement, portant atteinte à la sincérité et clarté de ces débats : comment peut-on organiser un débat sur une motion de censure si celle-ci n'a pas été réellement déposée et n'est pas consultable?

${ }^{10}$ Le calendrier des consultations prévoit une consultation des groupes parlementaires mineurs et des Présidents d'Assemblées le 21 août (Groupes des Autonomies SVP-PATT,UV du Sénat; Groupe mixte du Sénat et de la Chambre des députés ; Groupes parlementaires Liberi e Uguali, de la Chambre des députés) et groupes parlementaires majeurs (Groupe Fratelli d'Italia du Sénat et de la Chambre des députés; Groupe du Parti Démocrate du Sénat et de la Chambre des députés; Groupe de Forza Italia de la Chambre des députés et du Sénat ; Groupe de la Ligue, du Sénat et de la Chambre des députés ; Groupe du Mouvement Cinq Étoiles du Sénat et de la Chambre des députés).

Cfrhttps://www.quirinale.it/elementi/35136. 
cela le calendrier des consultations dessert cette finalité proposant deux possibilités : un nouveau gouvernement, des élections anticipées ${ }^{11}$. C'est ainsi qu'à l'issue des lères consultations ouvertes après la démission du Premier Ministre Conte, le Président de la République a décidé de donner plus de temps aux partis afin de pouvoir composer une nouvelle majorité gouvernementale, avec une échéance prévue pour le 27 août. A partir de cette date un 2ème tour de consultations (les 27 et $28^{12}$ ) a été organisé pour aboutir à deux solutions : un nouveau gouvernement ou bien de nouvelles élections. La solution retenue a été celle de redonner un nouveau mandat à Giuseppe Conte pour former un nouveau gouvernement avec une nouvelle majorité ${ }^{13}$. Les procédures mises en place témoignent d'une rationalisation des procédés des consultations, celle-ci est bien présente en observant le calendrier des consultations qui a constitué une clef de voûte importante pour la parlementarisation de cette crise et aussi pour sa résolution.

\footnotetext{
${ }^{11}$ A souligner qu'en cas d'élections anticipées cela aurait été la première fois dans l'histoire républicaine italienne qu'une législature dure moins de deux ans, contrairement à la XIème, XIIème et XVème législature.

${ }^{12} \mathrm{Cfrr}$. https://www.quirinale.it/elementi/35321

${ }^{13}$ Le Gouvernement Conte II constitue un cas singulier et sans précédent dans l'histoire constitutionnelle italienne. Pour la première fois on assiste à la naissance d'un nouveau gouvernement avec un alternance de forces politiques qui étaient majoritaires et d'oppositions au début de la XVIIIème législature : la Ligue qui était majoritaire lors du Conte I est passé ensuite à l'opposition du Gouvernement Conte II, et le Parti Démocrate à l'opposition du Conte I et, ensuite, force majoritaire du Gouvernement Conte II. On observe donc une alternance des forces politiques au Gouvernement, tout en gardant à la tête de ce dernier le même Premier Ministre. Généralement sous les précédentes législatures on assistait à des remaniements gouvernementaux qui consistaient à modifier l'équipe ministérielle, tout en gardant la même majorité politique (c'est le cas du Gouvernement d'Alema II pendant la XIIIème législature et Berlusconi III de la XIVème législature). La XVIIIème législature aura constitué un cas unique dans l'histoire républicaine italienne car un an à peine après les élections de 2018 se seront succédé au Gouvernement les deux forces politiques (Ligue, Parti Démocrate) indispensables pour former une majorité, en raison de l'absence de majorité à la fois à la Chambre des Députés et au Sénat.
} 